\title{
Homogeneous-heterogeneous reactions in boundary-layer flow of a nanofluid near the forward stagnation point of a cylinder
}

\author{
Qingkai Zhao \\ Collaborative Innovation Center for \\ Advanced Ship and Deep-Sea \\ Exploration(CISSE), \\ State Key Lab of Ocean Engineering, \\ School of Naval Architecture, \\ Ocean and Civil Engineering, \\ Shanghai Jiao Tong University, \\ Shanghai 200240, China \\ Email: qkzhao@sjtu.edu.cn
}

\author{
Hang $\mathbf{X u}^{*}$ \\ Collaborative Innovation Center for \\ Advanced Ship and Deep-Sea \\ Exploration(CISSE), \\ State Key Lab of Ocean Engineering, \\ School of Naval Architecture, \\ Ocean and Civil Engineering, \\ Shanghai Jiao Tong University, \\ Shanghai 200240, China \\ Email: hangxu@sjtu.edu.cn
}

\author{
Longbin Tao \\ School of Marine Science and Technology, \\ Newcastle University, Newcastle NE1 7RU, U.K. \\ Email: I.tao@ncl.ac.uk
}

\begin{abstract}
A mathematical model describing the homogeneousheterogeneous reactions in the vicinity of the forward stagnation point of a cylinder immerged in a naofluid is established. We assume that the homogeneous reaction is given by isothermal cubic autocatalator kinetics, while the heterogeneous reaction is chosen as first order kinetics. The existence of multiple solutions through hysteresis bifurcations is discussed in detail for the various diffusion coefficients of reactant and autocatalyst.
\end{abstract}

\section{Introduction}

The homogeneous and heterogeneous reactions are often encountered in many chemically reacting processes such as combustion, catalysis and biochemical systems. In such procedures, the homogenous reaction take places in the bulk and the heterogeneous reaction occurs on the catalyst surface. A common assumption for this flow problem is that the reaction only takes place in the boundary layer region of a body surface with an external flow being imposed on the outer edge of the boundary layer [1-3]. The problem can thus be simplified by using the boundary layer flows, usually stagnation-point flows [4-6]. Several experimental studies on methane/ammonia and propane oxidation flow-

*Address all correspondence related to ASME style format and figures to this author. ing over platinum have been given by Williams and his collaborators $[7,8]$, which clearly exhibit the existence of such homogeneous-heterogeneous reactions. Some theoretical works have been done to testifying the maturity of this model by some researchers [9-12].

Recently, nanofluids received considerable attractions owing to their better heat transfer capabilities. Several mathematical models have been proposed to predict nanofluids' behaviours such as the homogenous flow model [13], the dispersion model [14], the Buongiorno's model [15]. Among those models, the Buongiorno's model [15] received applausively attention since it well explained the slip mechanisms between the nanoparticles and the base fluid. In literature, few researchers have considered the homogeneous and heterogeneous reactions in nanofluids. Until now, only Kameswaran et al. [16] considered such chemical reactions in nanofluids. While their approach was based on the homogenous flow model, that was criticized by Magyari [17] that the governing equations of the homogeneous flow models can be reduced with the help of scaling transformations to the regular fluids. As a result, the corresponding nanofluid results can be recovered from the solutions of the Newtonian fluid problems via simple arithmetic operations. Different from the work of Kameswaran et al. [16], we use the Buongiorno's model [15] to model the nanofluid flow behaviours in the stagnation-point region of a two-dimensional body in the presence of the homogeneous-heterogeneous reactions. 
As far as we know, few studies have been done on such homogeneous-heterogeneous reaction problems in nanofluids by this model so that our work is unique and original.

\section{Mathematical descriptions}

Inspired by Merkin [18], we develop a model on boundary layer flow of a nanofluid in the forward stagnation-point region of a two-dimensional cylinder in the presence of the homogeneous and heterogeneous reactions. The sketch of this problem is shown in Fig.1. In the forward stagnation point, we assume that the reaction in the bulk is isothermal cubic autocatalytic, given by

$$
A+2 B \rightarrow 3 B, \quad \text { rate }=k_{1} C_{a} C_{b}^{2},
$$

while on the catalyst surface we have the single, isothermal, first-order reaction

$$
A \rightarrow B, \quad \text { rate }=k_{s} C_{a} .
$$

Here it is assumed that there is only reactant $A$ in the external flow, which has a uniform concentration $C_{\infty}$. Due to Eq.(1), we assume that the reaction rate is zero at the outer edge of the boundary layer. Based on those assumptions, the governing equations for boundary layer flow of a nanofluid in the forward stagnation-point region are written by

$$
\begin{aligned}
& \frac{\partial u}{\partial x}+\frac{\partial v}{\partial y}=0, \\
& u \frac{\partial u}{\partial x}+v \frac{\partial u}{\partial y}=-\frac{1}{\rho} \frac{\partial p}{\partial x}+v\left(\frac{\partial^{2} u}{\partial x^{2}}+\frac{\partial^{2} u}{\partial y^{2}}\right), \\
& u \frac{\partial T}{\partial x}+v \frac{\partial T}{\partial y}=\alpha\left(\frac{\partial^{2} T}{\partial x^{2}}+\frac{\partial^{2} T}{\partial y^{2}}\right)+\tau D_{B}\left(\frac{\partial C_{b}}{\partial x} \frac{\partial T}{\partial x}\right. \\
& \left.+\frac{\partial C_{b}}{\partial y} \frac{\partial T}{\partial y}\right)+\tau \frac{D_{T}}{T_{\infty}}\left[\left(\frac{\partial T}{\partial x}\right)^{2}+\left(\frac{\partial T}{\partial y}\right)^{2}\right] \\
& u \frac{\partial C_{a}}{\partial x}+v \frac{\partial C_{a}}{\partial y}=D_{A}\left(\frac{\partial^{2} C_{a}}{\partial x^{2}}+\frac{\partial^{2} C_{a}}{\partial y^{2}}\right)-k_{1} C_{a} C_{b}^{2} \\
& u \frac{\partial C_{b}}{\partial x}+v \frac{\partial C_{b}}{\partial y}=D_{B}\left(\frac{\partial^{2} C_{b}}{\partial x^{2}}+\frac{\partial^{2} C_{b}}{\partial y^{2}}\right) \\
& +\frac{D_{T}}{T_{\infty}}\left(\frac{\partial^{2} T}{\partial x^{2}}+\frac{\partial^{2} T}{\partial y^{2}}\right)+k_{1} C_{a} C_{b}^{2} .
\end{aligned}
$$

subject to the boundary conditions

$$
\begin{aligned}
& u=0, \quad v=0, \quad T=T_{w}, \quad D_{A} \frac{\partial C_{a}}{\partial y}=k_{s} C_{a}, \\
& D_{B} \frac{\partial C_{b}}{\partial y}=-k_{s} C_{a} \quad \text { at } \quad y=0, \\
& u \rightarrow u_{e}=a x(a>0), \quad T \rightarrow T_{\infty}, \quad C_{a} \rightarrow C_{\infty}, \\
& C_{b} \rightarrow 0 \quad \text { as } \quad y \rightarrow \infty .
\end{aligned}
$$

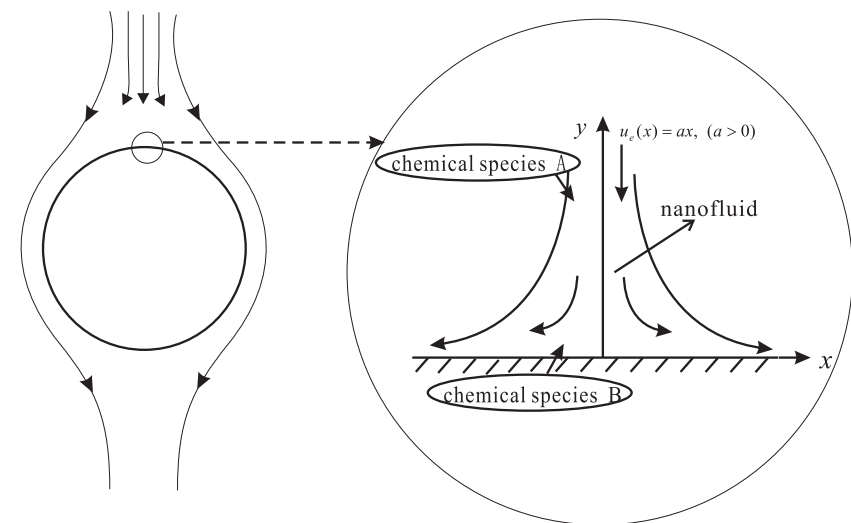

Fig. 1. Physical model and coordinate system

Within the boundary layer assumptions, we introduce the following similarity transformations and nondimensional quantities

$$
\begin{aligned}
& \psi(x, y)=(a v)^{1 / 2} x f(\eta), \quad u=a x f^{\prime}(\eta), \\
& v=-\frac{\partial \psi}{\partial x}=-(a v)^{1 / 2} f(\eta), \quad \theta(\eta)=\frac{T-T_{\infty}}{T_{w}-T_{\infty}}, \\
& \xi(\eta)=\frac{C_{a}}{C_{\infty}}, \quad \phi(\eta)=\frac{C_{b}}{C_{\infty}}, \quad \eta=\left(\frac{a}{v}\right)^{1 / 2} y .
\end{aligned}
$$

into Eqs.(3)-(7), the continuity equation(3)is satisfied automatically, and other equations are reduced to

$$
\begin{aligned}
& f^{\prime \prime \prime}+f f^{\prime \prime}+1-f^{\prime 2}=0, \\
& \frac{1}{P r} \theta^{\prime \prime}+N b \phi^{\prime} \theta^{\prime}+N t \theta^{\prime 2}+f \theta^{\prime}=0, \\
& \frac{1}{S c_{A}} \xi^{\prime \prime}+f \xi^{\prime}-K \xi \phi^{2}=0, \\
& \frac{1}{\varepsilon S c_{A}}\left(\phi^{\prime \prime}+\frac{N t}{N b} \theta^{\prime \prime}\right)+f \phi^{\prime}+K \xi \phi^{2}=0 .
\end{aligned}
$$

subject to the boundary conditions

$$
\begin{aligned}
& f^{\prime}(0)=0, \quad f(0)=0, \theta(0)=1, \\
& \xi^{\prime}(0)=K_{s} \xi(0), \phi^{\prime}(0)=-\varepsilon K_{s} \xi(0), \\
& f^{\prime}(\infty)=1, \theta(\infty)=0, \xi(\infty)=1, \phi(\infty)=0,
\end{aligned}
$$

where

$$
\begin{aligned}
& \operatorname{Pr}=\frac{v}{\alpha}, \quad N b=\frac{\tau C_{\infty} D_{B}}{v}, N t=\frac{\tau D_{T} \Delta T}{v T_{\infty}} S c_{A}=\frac{v}{D_{A}} \\
& \varepsilon=\frac{D_{A}}{D_{B}}, \quad K=\frac{k_{1} C_{\infty}^{2}}{a}, \quad K_{S}=\frac{k_{S}}{D_{A}}\left(\frac{a}{v}\right)^{-1 / 2} .
\end{aligned}
$$

\section{Results}

In this part, the solution diversities are examined in detail. In the computation, we assume that the diffusion coefficients of chemical species $A$ and $B$ are of a comparable size, 
which is not against real circumstance. In addition, the momentum equation and the energy equation have been studied by other researchers $[19,20]$. And note that we can use the approximate relation $C_{a}+C_{b}=1$ to get all results for $\phi(\eta)$, we therefore only consider $\xi(\eta)$ in this note.

In this paper, the reduced governing equations(11)-(14) with the boundary conditions(15) are solved by means of the shooting method associate with fourth order Runge-Kutta Scheme. The step size $\Delta \eta$ has to be adjusted for different values of the parameters to maintain the necessary accuracy. In our study, the value of $\Delta \eta$ between 0.001 and 0.01 were used, in order that the numerical values obtained are mesh in-dependent. On the other hand, a uniform grid of $\Delta \eta=$ 0.001 was found to be satisfactory for a convergence criterion of $10^{-8}$ which gives accuracy to seven decimal place, in nearly all case.

As shown in Fig.2, the multiple solutions can be in existence for particular sets of the heterogeneous reaction rate $K_{s}$ and the homogenous reaction rate $K$. As $K_{s}$ is considerably small, the multiple solutions are possible for some values of $K$. When $K_{S}$ increases gradually, the multiple solutions vanish. In other words, the range of $K$ for existence of multiple solutions begins to appear and grows significantly as $K_{S}$ continuously diminishes. Particularly, for $K=0$, we notice that the maximum value of $\xi(0)$ approaches to 1 as $K_{s}$ becomes negligibly small. We then examine the influence of the ratio of $N_{t} / N_{b}$ on solution multiplicities. As shown in Fig.3, $N_{t} / N_{b}$ can alter solution behaviour distinctly. For example, at $K_{s}=0.025$, the multiple solutions could be possible (see Fig.2). While as $N_{t} / N_{b}$ increases to a sufficiently large value, the multiple solutions start to be disappeared. We also notice that the maximum value of $\xi(0)$ increases as $N_{t} / N_{b}$ enlarges. It is worth noting that we choose the parameter $\varepsilon=$ 1 in Fig.3. When $N_{t} / N_{b}=0$, our problem is simplified to Chaudhary and Merkin's work [2], and we can find that our figure is the same as Fig.1(c) of Chaudhary and Merkin's

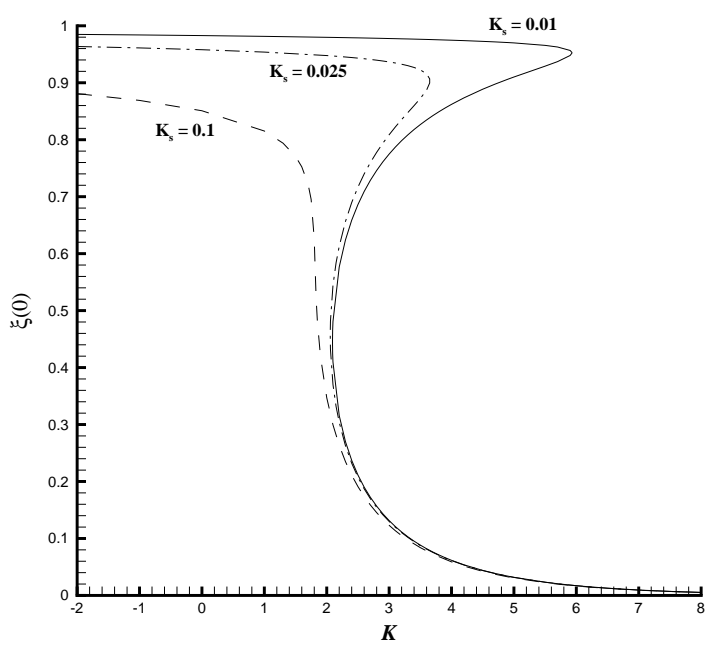

Fig. 2. $\xi(0)$ against $K$ with $P r=1, N b=1, N t=0.05, S c=1$, $\varepsilon=1.5$ for some values of $K_{s}$.

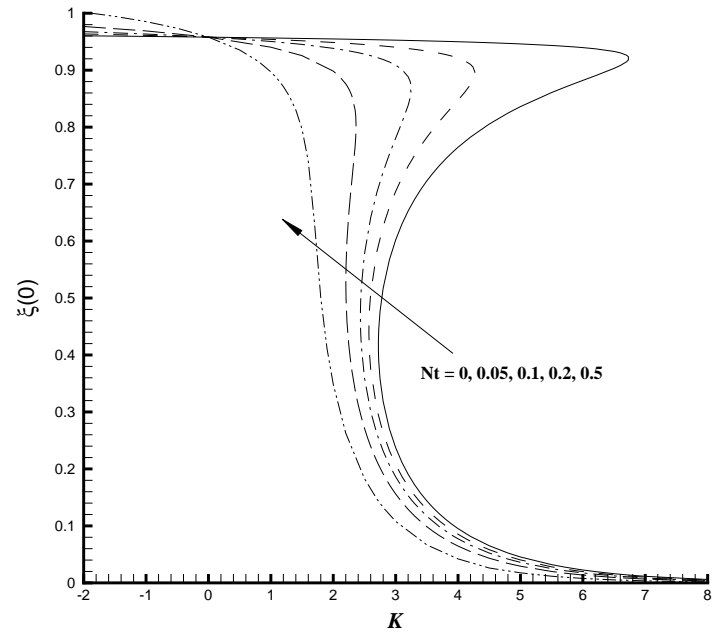

Fig. 3. $\xi(0)$ against $K$ with $P r=1, N_{b}=1, K_{s}=0.025, S c=1$, $\varepsilon=1$ for different values of $N_{t}$.

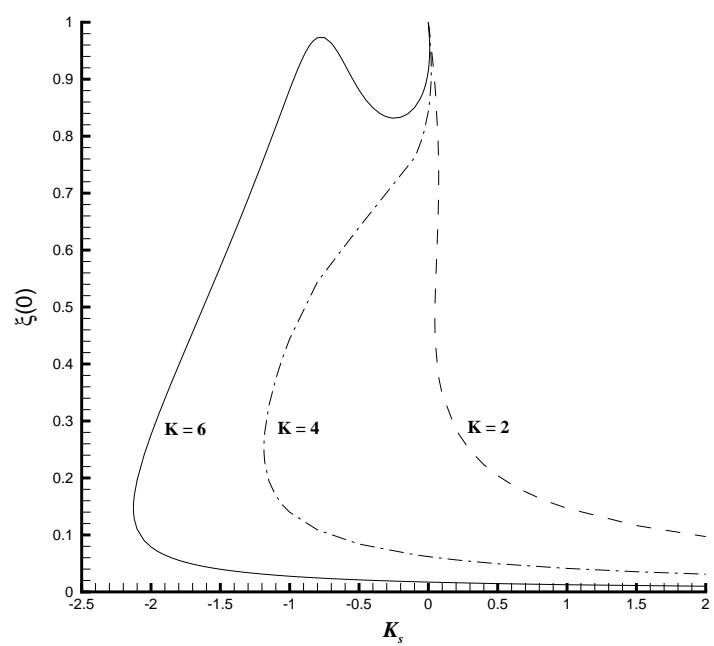

Fig. 4. $\xi(0)$ against $K_{s}$ with $P r=1, N b=1, N t=0.05, S c=1$, $\varepsilon=1.5$ for different values of $K$.

work in the case $K_{s}=0.025$.

Fig.4 exhibits the relation of $K$ and $K_{S}$ on solution diversifies. It is found that as the homogeneous reaction rate is week enough, no multiple solutions are valid for any $K_{s}$. While as this value is considerably large, the solution bifurcations begin to appear. Note that the heterogenous reaction rate $K_{s}$ must be greater than or equal to zero to meet physical requirements. It is found from Fig.5, the ratio of the diffusion coefficient $\varepsilon$ has effects on solution behaviours. For prescribed values of $K$, the multiple solutions come out for small $\varepsilon$. When $\varepsilon$ becomes sufficient large, the multiple solutions vanish. We further notice that a tiny variation of $\varepsilon$ can causes a significant increase of $\xi(0)$ when $\varepsilon$ is sufficiently small. As $\varepsilon$ is considerably large, $\xi(0)$ diminishes rapidly as 


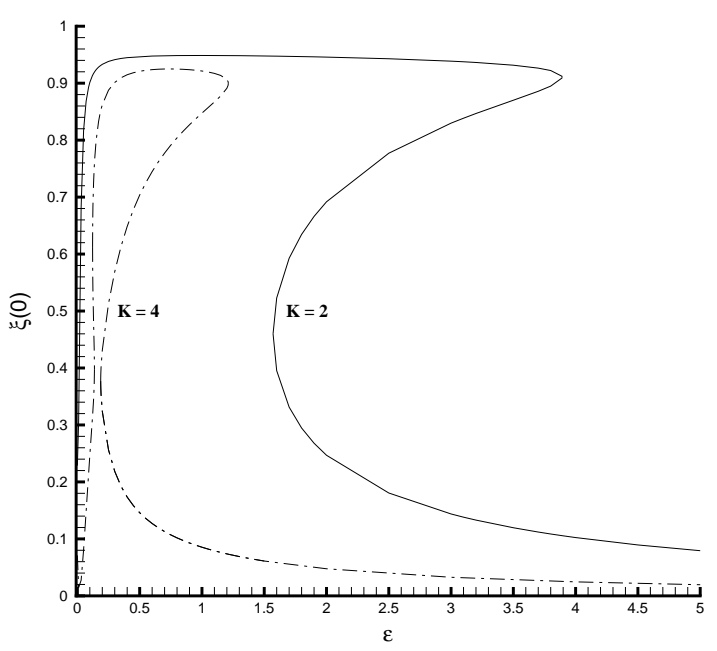

Fig. 5. $\xi(0)$ against $\varepsilon$ with $\operatorname{Pr}=1, N_{b}=1, N_{t}=0.05, S c=1$, $K_{s}=0.025$ for different values of $K$.

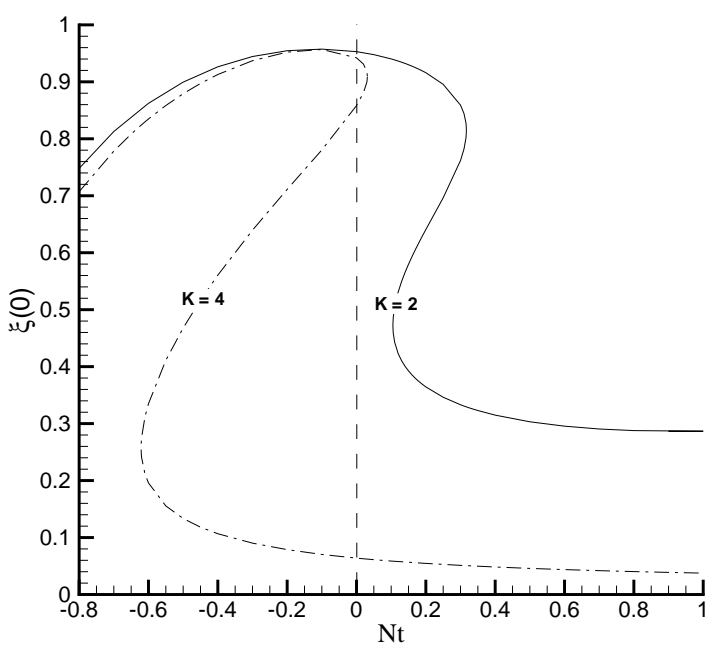

Fig. 6. $\xi(0)$ against $N_{t}$ with $\operatorname{Pr}=1, S c=1, N_{b}=1, \varepsilon=1.5$, $K_{s}=0.025$ for different values of $K$.

$\varepsilon$ evolves. Fig. 6 illustrates the effect of $N_{t} / N_{b}$ on $\xi(0)$. Similarly for some values of $N_{t} / N_{b}$, the multiple solutions are possible. While physically, $N_{t} / N_{b}$ must be positive. Hence only dual solutions that could be physically realistic is expected for a range values of $N_{t} / N_{b}$. As $N_{t} / N_{b}$ continuously grows, the multiple solutions disappear and only one solution can be found.

From the perspective of physical significance, we note that, the values of $K, K_{s}$ and $N t / N b$ have to be nonnegative. Moreover, the concentrations of $C_{a}$ and $C_{b}$ cannot be greater than 1 . As a result, solutions in this range are physically realistic. On the other hand, as suggested by Chaudhary and Merkin [2, 3], for large value of $K$, the solution represents that the homogenous reactions are dominant. When $K$ is s- mall and $K_{s}$ is positive, the heterogeneous (surface) reactions become important. We therefore can conclude both solutions are meaningful as all parameters are chosen physically realistic. In some special cases, for example $K=0$, there is no homogeneous reaction, the system can still sustain a surface reaction with the concentration of reactant $\mathrm{A}$ having a value which below the ambient value $C_{\infty}$. When $K_{s}=0$, there is no heterogeneous reaction on the surface, the homogeneous reaction is sufficiently strong to be self-sustaining even when there is a loss of reactant from the surface. This corresponds to having an endothermic homogeneous reaction. In other cases, both homogeneous reaction and heterogeneous reaction exist.

\section{Conclusions}

The homogeneous-heterogeneous reactions in boundary-layer nanofluid flow near a forward stagnationpoint on a cylinder surface has been studied. The Chaudhary and Merkin's model [1] has been extended to nanofluids via Buongiorno's nanofluid model [15]. It is found that the homogeneous reaction rate parameter $K$, the heterogeneous reaction rate parameter $K_{s}$, the diffusivity ratio $\varepsilon$ and the ratio $N_{t} / N_{b}$ could leads to multiple solutions. In summary, the following conclusions can be reached:

1. The appropriate sets of $K$ and $K_{S}$ could produce multiple solutions. While solutions for negative $K_{s}$ have no physical meanings;

2. The diffusivity ratio $\varepsilon$ can affect solution behaviours to a large extent. For average values $\varepsilon$, triple solutions are expected;

3. $N_{t} / N_{b}$ can cause solution diversifies. While the negative value of $N_{t} / N_{b}$ has only mathematical meanings. Dual solutions can be found for positive values of $N_{t} / N_{b}$.

\section{Acknowledgements}

We warmly thank the very competent reviewers for their time spent on reading the manuscript and for the valuable comments and suggestions.

\section{Nomenclature}

A, B two different kinds of chemical species.

$C_{a}, C_{b}$ concentration of the chemical species A and B, respectively.

$C_{\infty}$ reference concentration of the chemical species $\mathrm{A}$ in external flow.

$D_{A}, D_{B}$ diffusion coefficient of chemical species A and B, respectively.

$D_{T}$ thermophoretic diffusion coefficient.

$f$ dimensionless velocity.

$k_{1}, k_{s}$ homogeneous and heterogeneous reaction rate constants, respectively.

$K$ homogeneous reaction rate parameter.

$K_{s}$ heterogeneous reaction rate parameter.

$\mathrm{Nb}$ Brownian motion parameter. 
$N t$ thermophoresis parameter.

$p$ pressure.

$\mathrm{Pr}$ Prandtl number.

$T$ temperature.

$u, v$ velocity components in $\mathrm{x}$ and $\mathrm{y}$ directions, respective$1 y$.

$u_{e}$ free stream velocity.

$x, y$ coordinates.

\section{Greek symbols}

$\alpha$ thermal diffusivity.

$\varepsilon$ ratio of the diffusion coefficient

$v$ kinematic viscosity.

$\mu$ dynamic viscosity.

$\rho$ fluid density.

$\tau$ ratio of the effective heat capacity of the nanoparticle to that of the fluid.

$\tau_{w}$ shear stress.

$\eta$ similarity variable.

$\theta(\eta)$ reduced temperature.

$\xi(\eta)$ reduced concentration of chemical species A.

$\phi(\eta)$ reduced concentration of chemical species B.

$\psi$ dimensionless stream function.

\section{References}

[1] M.A. Chaudhary, J.H. Merkin, Free-convection stagnation-point boundary layers driven by catalytic surface reactions: I the steady states. J. Eng. Math., 28(2) (1994) 145-171.

[2] M.A. Chaudhary, J.H. Merkin, A simple isothermal model for homogeneous-heterogeneous reactions in boundary-layer flow. I Equal diffusivities. Fluid Dyn. Res., 16 (1995) 311-333.

[3] M.A. Chaudhary, J.H. Merkin, A simple isothermal model for homogeneous-heterogeneous reactions in boundary-layer flow. II Different diffusivities for reactant and autocatalyst. Fluid Dyn. Res., 16 (1995) 335359.

[4] X. Song, W. R. Williams, L. D. Schmidt, R. Aris, Bifurcation behavior in homogeneous-heterogeneous combustion: II. Computations for stagnation-point flow. Combust. Flame, 84(3-4) (1991) 292-311.

[5] Song, X., L.D. Schmidt, R. Aris, Steady states and oscillations in homogeneous-heterogeneous reactions systems. Chem. Eng. Sci. 46 (1991) 1203-1215.

[6] H. Ikeda, P.A. Libby, F.A. Williams, Catalytic combustion of hydrogen-air mixtures in stagnation flows. Combust. Flame, 93 (1993) 138-148.

[7] W.R. Williams, M.T. Stenzel, X. Song, L.D. Schmidt, Bifurcation behavior in homogeneous-heterogeneous combustion. I. Experimental results over platinum. Combust. Flame, 84 (1991) 277-291.

[8] W.R. Williams, J. Zhao, L.D. Schmidt, Ignition and extinction of surface and homogeneous oxidation of $\mathrm{NH} 3$ and CH4. A.I.Ch.E. J., 37 (1991) 641-649.

[9] P.J. Ogren, Analytical results for first-order kinetics in flow tube reactors with wall reactions. J. Phys. Chem., 79(17) (1975) 1749-1752.
[10] V.D. Dang, M. Steinberg, Convective diffusion with homogeneous and heterogeneous reactions in a tube. $J$. Phys. Chem., 84(2) (1980) 214-219.

[11] K.M. Nigam, V.K. Srivastava, K.D.P. Nigam, Homogeneous-heterogeneous reactions in a tubular reactor: an analytical solution. J. Phys. Chem., 25(2) (1982) 147-150.

[12] C.Y. Chang, Y.J. Yang, C.F. Lin, Mixed convection and diffusion of reactants, products, and heat with arbitraryorder heterogeneous and homogeneous reactions in a rectangular duct. Int. J. Heat Transfer, 28 (1985) 1813 1821.

[13] S. U. S. Choi, Enhancing thermal conductivity of fluids with nanoparticles, in: Proceedings of the 1995 ASME International Mechanical Engineering Congress and Exposition FED 231/ MD66, ASME, San Francisco, CA, USA, 1995, pp. 99-105.

[14] Y.M. Xuan, W. Roetzel, Conceptions for heat transfer correlation of nanofluids. Int. J. Heat Transfer, 43(19) (2000) 3701-3707.

[15] J. Buongiorno, Convective transport in nanofluids. $J$. Heat Transfer ASME, 128(3) (2006) 240-250.

[16] P.K. Kameswaran, S. Shaw, P. Sibanda, P.V.S.N. Murthy, Homogeneous-heterogeneous reactions in a nanofluid flow due to a porous stretching sheet. Int. J. Heat Mass Transfer, 57(2) (2013) 465-472.

[17] E. Magyari, Comment on the homogeneous nanofluid models applied to convective heat transfer problems. Acta Mech., 222(3) (2011) 381-385.

[18] J. H. Merkin, A model for isothermal homogeneousheterogeneous reactions in boundary -layer flow. Mathematical and Computer Modelling, 24(8) (1996) 125136.

[19] N.Bachok, A.Ishak, I. Pop, On the stagnationpoint flow towards a stretching sheet with homogeneousCheterogeneous reactions effects. Communications in Nonlinear Science and Numerical Simulation, 16(11) (2011) 4296-4302.

[20] M.Mustafa, T. Hayat, I. Pop, S. Asghar, S. Obaidat, Stagnation-point flow of a nanofluid towards a stretching sheet. International Journal of Heat and Mass Transfer, 54(25) (2011) 5588-5594. 